\title{
An Optimal Energy Consumption Based Resource Management in Mobile Cloud Computing
}

\author{
M. R. Sudha, C. P. Sumathi, A. Saravanakumar
}

\begin{abstract}
This paper focused on optimal energy-efficient resource allocation management in the mobile cloud services. A resource management technique depicts the various resources reservation or blocking. Energy wastage is diminished, and revenue is amplified for mobile cloud providers. The recommended method holds two stages: a) beginning stage, the task impairment, delay time, resource utilization for every task has been individually calculated, and the enthalpy was measured, and b) the second stage, the enthalpy-based Optimal Energy Allocation Supervision (OEAS) algorithm was used to optimize the resources to the powerful resource management. In this paper, the problem of the limited and relatively small battery energy power in today's mobile devices has been restricted functionality which can include into these platforms. Diverse mobile cloud suppliers helpfully share the resources in a pool for improving resource allocation based on the users demand and distribute revenue in mobile cloud providers. The recent upgraded research in MCC from an existing work has been examined on the issues of managing resources and vital challenges in energy consumption. The new hazing technology of mobile users and robust business interests in mobile cloud environment which escort the innovative progress in mobile cloud computing. It operates intense energy methods with a low cost. This paper exhibits the research extent and classified various issues in energy saving in mobile clouds. Later, it analyzes the presented research results and mechanisms which establish its strengths and weaknesses. Energy consumption is a major problem being faced by mobile cloud computing. This paper recognizes and explains the open issues and idea of future research. The main objective is to reduce energy consumption, increase energy efficiency in computing devices and resource allocations management as well as in their executions. Energy conservation can be the optimal solution which is minimized by using less of an energy service.
\end{abstract}

Index Terms: Energy Resource Allocation, Mobile Cloud Computing, OEAS Algorithm

\section{INTRODUCTION}

Mobile cloud has been evolved from the combination of both mobile and cloud platforms [1]. It is a recent technical innovative paradigm for strengthening mobile cloud users.

Mobile cloud computing promote the concept of mobile applications, data handling in data centers in the cloud. The

Revised Manuscript Received on July 05, 2019.

M. R. Sudha, Department of Computer Applications, Faculty of Science and Humanities, SRM Institute of Science and Technology, Kattankulathur - 603203, Chennai, Tamil Nadu, India. sudham2@srmist.edu.in

Dr. C. P. Sumathi, Department of Computer Science, S. D. N. B. Vaishnav College for Women Name, Chromepet, Chennai, 600 044, Tamil Nadu, India.drcpsumathi@gmail.com

Dr. A. Saravanakumar, Department of Physics, Easwari Engineering College, Ramapuram, Chennai - 600 089, Tamil Nadu, India.sara_mnes@yahoo.co.in mobile cloud computing reduces the execution time of mobile applications and minimizes the energy consumption of mobile devices [2]. The technique of allocating resources wishes to maintain the service quality (QoS) and energy efficiency. These are essential to get maximum resource utilization. To do this task, the resource pool has produced.

The main advantage of Mobile cloud computing is an ordered data storage and processing in remote mobile devices. A mobile cloud application transfers the data from mobile devices into the cloud. It is a new pattern of the data processing and storage computing in mobile clouds [3]. These are centralized applications which can be accessed over the wireless local area network like mobile browser. The profits brought by Mobile Cloud Computing which have been checked and verified as future mobile applications [4]. It refers to an emerging trend in a mobile device from which an application launched. It makes it simple way of data handling in the mobile cloud.The resource provisioning is a process of allocating, managing and controlling required resources like CPU, Memory, I/O Devices, Files etc. and which might be the main issue of mobile services [5, 6].The mobile users have experienced issues like low battery, offloading data in less memory and processing speed. The performance of MCC is evaluated by means of the resource allocation with varying QoS factors [7] such as availability, throughput, protection, response time, reliability and efficiency. Fig 1 gives the Mobile cloud computing architecture wherein the mobile users access the mobile cloud services directly from their applications or through mobile service provider using their mobile data.

Cloud computing offer three layered services like SaaS (Software as a Service), PaaS (Platform as a Service) and IaaS (Infrastructure as a Service) in collaboration with RaaS (Resource as a Service) [8]. It deals with many issues of how to manege resources, and migration of resources to mobile users. It is well predictable as essential harms, such as inhibited computing power mainly limited battery life [9]. These scarce resources are hard to support assessment of precise applications, for instance video gaming, image compression, and social Medias [10]. H.Viswanathan et al. [11] represented a resource provisioning Mechanism for arranging the assorted devices in the environs. 


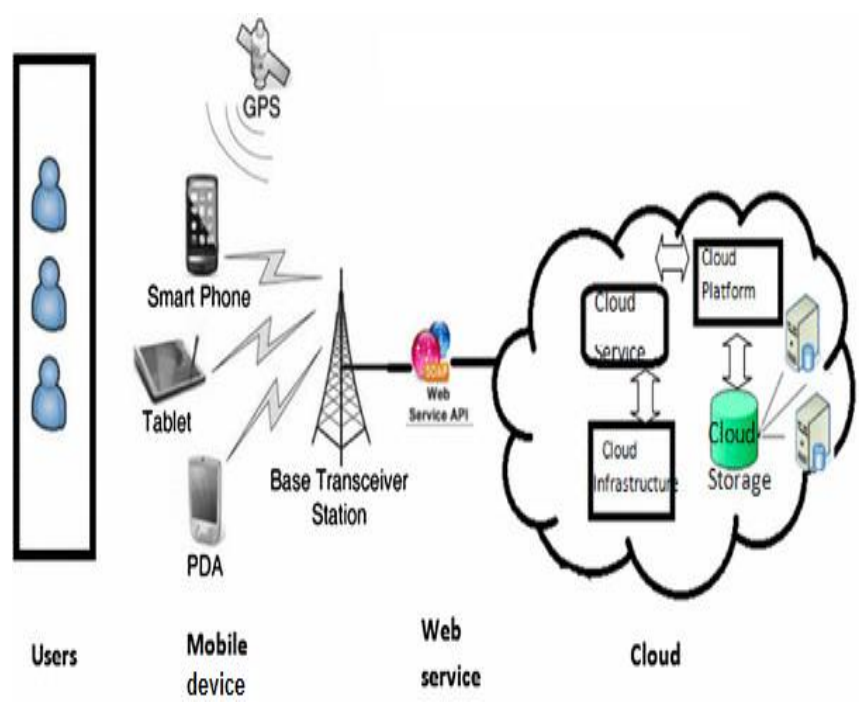

Fig 1. Mobile cloud computing architecture

Integration of the optimization of the resource is discussed in [12] for the energy inhibited mobile users solving the problem of distribution delay and energy conservation. Satyanarayanan et al., [13] developed a model which replaces the secluded cloud with nearby cloudlets. Zhang et al. introduced offloading concept of energy consumption of mobile devices [14]. The policy of Resource allocation is done by reserving resources which improves quality of service (QoS). It is vital for mobile cloud applications with real-time applications. The proficiency is the achieved by the maximum utilization of resource. To attempt this, resource pool can be produced. The advancements of execution of mobile cloud with a tremendous amount of energy consumption are discussed in T. Liu et al [15]. It outcomes with several challenges and solutions with managing resource strategy which give surety scalability, manageability, versatility, efficiency, and dependability of mobile cloud computing $[16,17]$. The resource allocation of MCC is a great challenging to face while estimating the relative size of the infrastructures [18, 19]. Few investigations have discussed these issues earlier [20-22]. Consequently, it is a prominent issue for service providers to design resource management systems in real time applications [23].

Benefits of MCC:

1. Minimal weight on the mobile resources leads to absolute battery life. Hence Users desire apps that are light on battery.

2. Use of mobile cloud applications are consequences in the better data storage capacity and high processing power.

3. Data is easily coordinated across multiple devices leading to quicker and simpler relocation and cross-device use.

4. While storing data on the cloud it relics secured though the device is damaged. By using password curls this data can also be protected ensuring user privacy.

5. The application runs smoothly on any mobile device. Users perceptibly prefer applications of fast, scalable and secure.
6. Improves reliability with information back up and stored in the cloud.

7. Applications use only the desired resources.

8. Mobile is connected to mobile cloud services through API architecture.

9. High rate and elasticity.

10. Mobile cloud applications can be built quickly by means of mobile cloud services.

11. Virtualization method make mobile cloud platform simply easy to control the resources.

12. It facilitates resource sharing, high utilization, swift provisioning and workload seclusion.

13. The current trends in virtualization are consolidation of data centers consequently with the minimal cost.

The aim is focused on the development of an optimal resource allocation policy through maximum system reward with high throughput and energy conservation.

This paper has explained as follows: Section 2 problem formulation of the related works regarding the recommended method, while Sections 3 provided an energy efficient resource allocation of the suggested methodology. Section 4 explained the Optimal Energy Allocation Supervision Algorithm conducted in this study, while Section 5 results discussed and finally Section 6 has concluded.

\section{PROBLEM FORMULATION}

The Mobile cloud computing challenges are based on three factors as true:

(1) Mobile broadband connectivity and high speed

(2) As mobile devices usage is increased, making an elegant MCC to provide the desired resources.

(3) Develop mobile cloud computing contribute resources to users at low cost and on-demand.

\section{A. Energy Conservation System}

Let assume system with a finite number of processors operated on mobile cloud computing. Each processor has different speeds but is assumed to be unary, i.e., each processor executes one task at a time. When other tasks are ready to execute, it requires the processor but it is not available at that time and so the process will be put into the Ready queue. The drawback is the cost and delay in activity execution. Here, the central focus is on allocating resources and reduces power consumption.

Resources can be allocated to the demanded nodes only. Thus switches off idle nodes 
and reduces power conservation.

\section{B. Green Mobile Cloud Design Model}

Designing the Next generation of Mobile Cloud Dat: Center with QoS is to create a model which includes fous things like consumers, Green cloud service allocator, Energy

1. Consumers: Originates from the service request and clouc consumer deploys a web service that is accessed by user. between the cloud infra structure and consumers.

3. Energy Monitor: It can be monitored by making resource allocation decisions with energy consumption.

4. Service Scheduler: It allocates the requested resource from the task and is responsible to add or remove the reques from it.

\section{ENERGY EFFICIENT RESOURCE ALLOCATION}

CPU is the heart of mobile cloud system. It consume large amount of energy in mobile cloud when it executes any task Memory is the next power consuming unit in computes system. During the execution time, memory requires very large amount of energy. Multi core CPUs are very power efficient in MCC. Energy efficient memory technologies use offloading mechanism.

This proposed model produces efficient energy usage in CPU. It is obtained by the operative mechanism with varying frequencies during the task computation. This research work concerns varying frequency scaling of CPU as its foundation to attain high energy efficiency. Moreover, it also regard as energy consumption during memory access despite the fact that making the offloading assessment. This work also recommends a methodology for estimating the energy consumption of tasks by considering processor and memory usage. This model also presents a dominant feasible energy efficient resource management technique with two stages as follows:

In the first stage, various Activities (task) measures were taken and based on the quantity the enthalpy calculated.

In the second stage, the enthalpy based Optimal Energy Allocation Supervision (OEAS) algorithm was proposed for the dominant feasible energy resource management scheme.

At this point, the (OEAS) algorithm pre-defined the number of activities based on the most favorable values for the assigned resources in the mobile cloud. Based on the usage of high enthalpy values, the priority can be calculated for job scheduling. The pictorial representation of the recommended approach depicted in Fig 2. monitor and Service scheduler.

2. Mobile cloud service allocator: It acts as a mediato

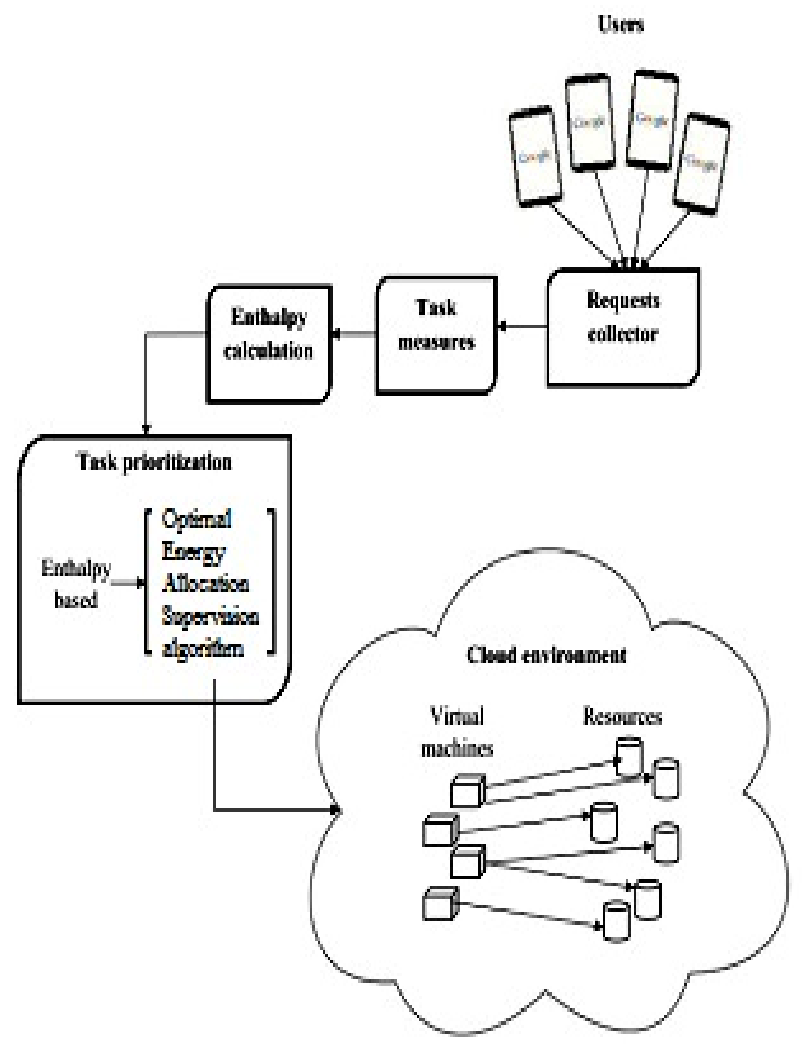

Fig 2. Mobile cloud environment

\section{A. Powerful Energy Efficient Resource Management}

In a dynamic mobile cloud platform, multiple service requests is coming from various users so that every order of demand request is made up of unique assigned activity. In a mobile cloud computing atmosphere, the mobile cloud server is the mobile cloud provider which handle the jobs and for every activity, unique values like task loss, waiting time, transmission outcomes, resource utilization rate and enthalpy value calculated for efficient resource management system. At this point, the mobile cloud owner has a major role in the resource management technique because it makes the decision of assigning the priorities among the multiple users.

\section{B. Task Measures Calculation}

In the resource allocation method, congestion may happen when more than one tasks wants to run at the same time. To avoid this situation, a powerful resource allocation technique is applied. In this paper, different task measures like task fault, waiting time, energy consumption, resource utilization and enthalpy was calculated as below.

\section{Task Fault}

The task fault was attained by the number of activities or task in the scheduling system and the number of scheduled tasks by using Equation (1),

$$
T_{L}=\frac{T(n)-T(m)}{T(m)}
$$

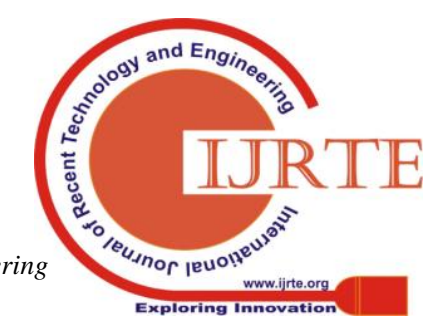


where $T(n)$ are the total number of activities in the system, $\mathrm{T}(\mathrm{m})$ is the number of scheduled tasks, where as TL is the task fault.

\section{Waiting Time}

Few packets that pass through a data center may not be reached on their destination due to link errors. This loss of data may cause salient communication delays. The delay was represented as time slot for completion of an activity. The delay is calculated by the time interval between the submissions to completion of task. Let assume the extra time taken by the activity to complete its entire process contains waiting time $(\mathrm{Wt})$ and the transfer time $(\mathrm{Tr})$, the delay time (Dt) by means of equation 2 , while the transfer time is calculated by using equation 3 and the waiting time, by equation 2 .

$$
\begin{gathered}
D_{t}=W_{t}+T_{r} \\
T_{r}=\stackrel{D_{t}}{B_{w}}
\end{gathered}
$$

where $\mathrm{D}$ represents each task delay time and $\mathrm{B}_{\mathrm{w}}$ is the bandwidth per task. $B_{w}$ is calculated by using equation 4 ,

$$
\mathrm{B}_{\mathrm{w}}=\frac{\mathrm{B}_{\mathrm{tot}}}{\mathrm{N}}
$$

where $\mathrm{B}_{\text {tot }}$ is the total bandwidth and $\mathrm{N}$ is the total number of requests. Time delay is the sum of all tasks waiting time which is found by equation (5)

$$
\mathrm{T}_{\mathrm{d}}=\mathrm{T}_{\text {wait }}
$$

where $T_{d}$ is the delay time, $T_{\text {wait }}$ is the sum of all tasks waiting time

\section{E. Energy Consumption}

The main objective is computing the power consumption of a point in a network. It is to avoid delays in the networks recital reduces power and thus minimize cost. When the networks are idle, power was released and the remaining energy for the packets and the equation represents the power spent in the computations that occur within the routing networks and power tunings. The power consumed by a network within time $\mathrm{t}$ is estimated by using the below equation,

$$
\mathrm{Ec}(\mathrm{t})=(\mathrm{NT} X \mathrm{~A})+(\mathrm{NR} X \mathrm{~B})
$$

where $E c(t)$ denotes the absorbed energy by the network after time t, NT and NR stands for the number of transmitted and received packets via the Net, $\mathrm{A}$ and $\mathrm{B}$ are constant factors .

\section{F. Enthalpy Measure}

Enthalpy is an energy oriented property or state function which has the dimensions of energy .In thermodynamics it can be defined as, "Enthalpy" is a measure of heat transfer". It does not refer to a process. Enthalpy is found by mathematical formula as shown below:
$\mathrm{H}=\mathrm{U}+\mathrm{PV}$

At this point, the enthalpy was determined by using the task measures. The enthalpy is determined by

$\mathrm{H}=\mathrm{Rv}(\mathrm{Rq})+\mathrm{PV}$

$\mathrm{PV}=\left(\mathrm{T}_{\mathrm{L}} \mathrm{X} \mathrm{D}_{\mathrm{t}} \mathrm{X} \mathrm{U}\right)$

where $\mathrm{H}$ is the enthalpy of the system, $\mathrm{Rv}$ and $\mathrm{Rq}$ are the reputation, $D_{t}$ is the Delay time, $T_{f}$ is the task failure and $U$ is the utilization. The OEAS algorithm uses high enthalpy values. Each task has priority based on the enthalpy values. The estimated enthalpy values are applied as the input to the OEAS algorithm. The proposed OEAS algorithm helps the cloud admin to assign the priorities and allocate resources efficiently based on this priority number.

\section{OPTIMAL ENERGY ALLOCATION SUPERVISION ALGORITHM}

The OEAS is an algorithm which was simulated after the reproduction policy. It laid spawn at the fundamental base level in the nest of other host level. At this time, it makes use of the estimated maximum value of enthalpy $(\mathrm{H})$. The proposed pseudo code an OEAS algorithm as shown in Fig 3.

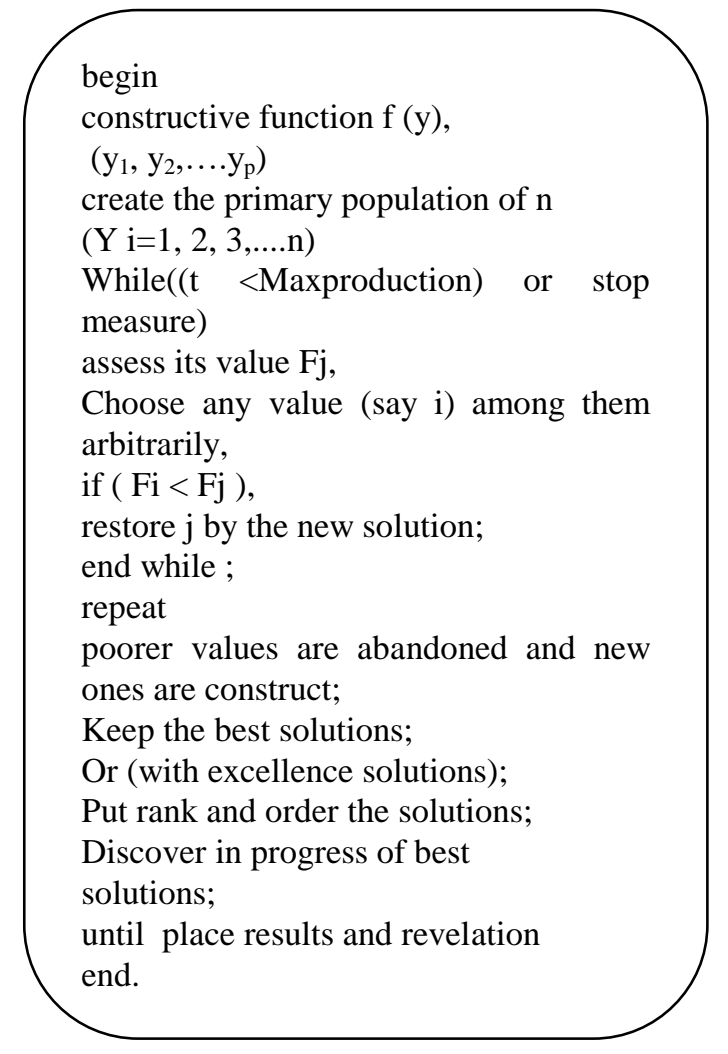

Fig 3. OEAS algorithm

Algorithm description/procedures

The pictorial representation of pseudo code (OEAS) algorithm is depicted in Figure 4. In the proposed algorithm, the resources are allocated based on the activity priority. This priority-based resource allocation resolved the issues in the distribution and ensured efficient store utilization. 
Resource allocation scheme is a main role in sharing computing resources among various clients. The resource allocation in data centers plays a vital role in both end-user satisfaction and cost of profit. The future extended work will formulate the mobile cloud service admin with choosing the priority for getting an efficient resource allocation. This resource allocation method is more effective than the previous ones. The mobile cloud admin may take decisions for task distribution based on the allocation of the resources. The OEAS algorithm will be completed with the optimal solution from the large number of iterations. In this method, the cloud owner can effectively allocate the resources to the end users with less wastage of energy and with maximum profit.

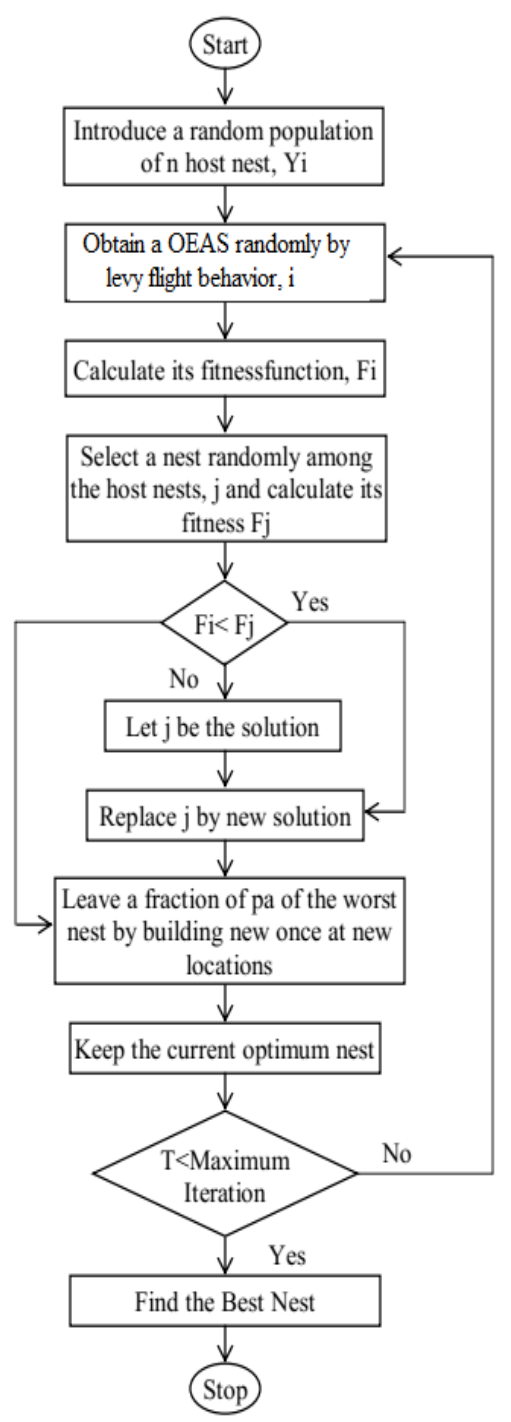

Fig 4. OEAS algorithm flowchart

\section{RESULTS AND DISCUSSION}

The suggested method in this study guarantees an efficient energy resource allocation management via the energy consumption and execution time. Often, their computational obscurity and computing capacity of processing elements were also minimized. The proposed methodology was executed on a MATLAB platform. The performance of the suggested technique was matched with that of other prevailing techniques.

\section{A. Performance Analysis}

Here, the performance of the suggested the method was evaluated by using various measures such as the execution time (by varying the nodes and iterations), energy loss (dynamic number of the nodes and iterations), and node loss (by changing the iterations and reputation) and benchmarking with the prevailing approaches.

\section{B. Execution Time}

The time interval between the submission of task and the completion is known as task execution time. This is estimated prior to the decision of whether to offload the task or not. The node and iteration based execution is obtained by the time of the recommended task with the previous one and the output is analyzed in Figure $5(\mathrm{a}, \mathrm{b})$. The outcome suggested task implementation time was less than that of the prevailing techniques.
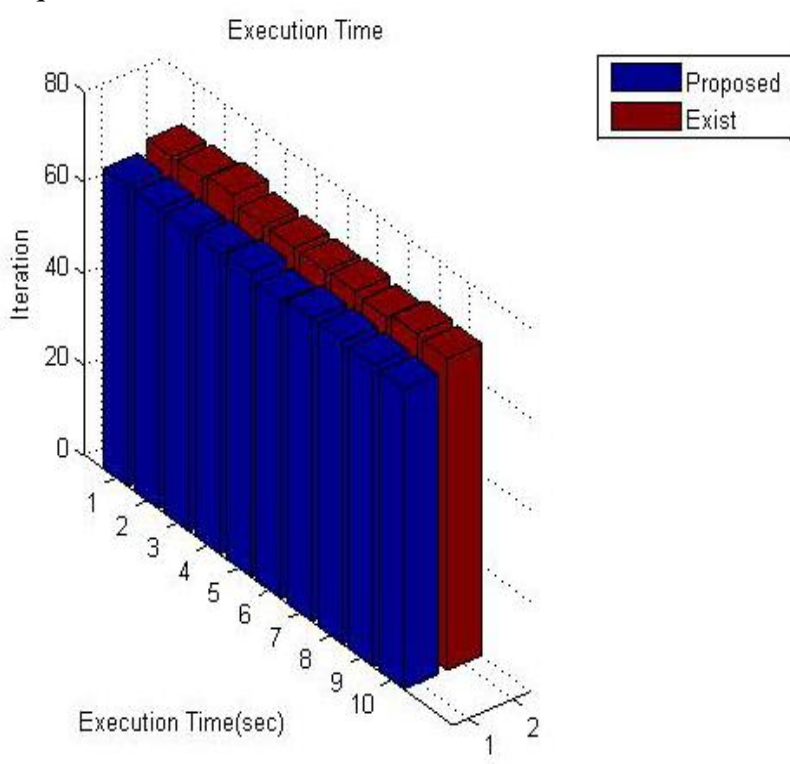

(a)

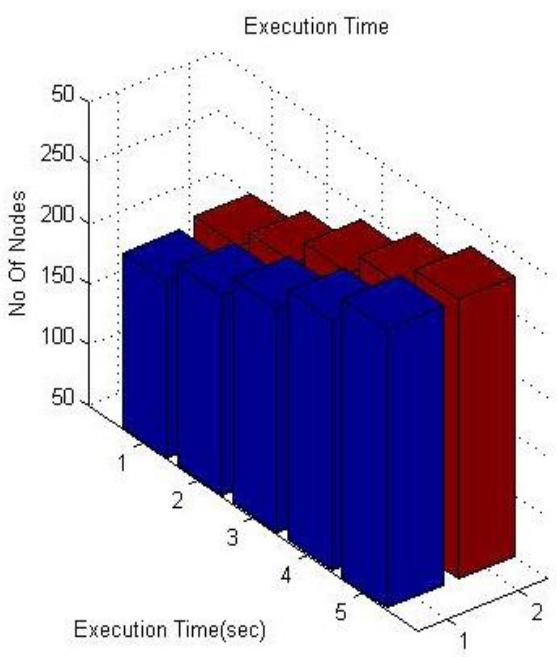

(b)

Fig 5. (a, b) Comparative analysis of OEAS with existing work for execution time

\section{Energy Loss}

Published By: 
The wastage of energy $\mathrm{E}_{\text {wastage }}$ is calculated using equation 10 ,

$\mathrm{E}_{\mathrm{wastage}}=\mathrm{F} \mathrm{X} \mathrm{T} \mathrm{X} \mathrm{P}_{\text {weight loss }}+\mathrm{T} \mathrm{X} \mathrm{P}_{\text {no weight loss }}$

where $E_{\text {wastage }}$ is the energy wastage, $F$ is the lose factor, $T$ is the time period, $\mathrm{P}_{\text {weight loss }}$ is the Power weight losses, $\mathrm{P}_{\text {no weight }}$ loss is the no-load losses.

\section{Delay}

The delay time is expressed as time taken for a data from origin to endpoint via internet. It is determined in units of multiples or fractions of a second. Delay time may vary based on the locality of the pair of interacting networks. The delay in the process execution time is directly relative to the length of the data. It is done by means of the below equation.

$\mathrm{T}_{\text {delay }}=\frac{\mathrm{N}}{\mathrm{R}}$ the message transfer rate. The communication delay is occurring in one way transmission. The proposed research work determines the time delay for different number of iterations which is mentioned in Figure 6.

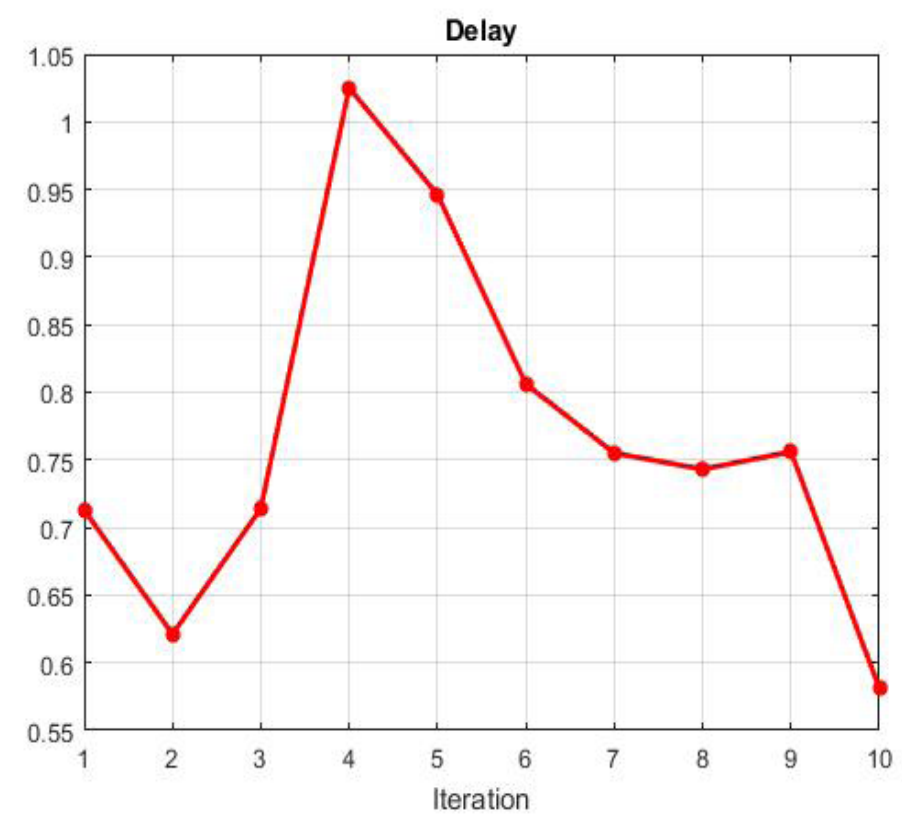

Fig 6. Delay by varying the iterations

In the upgrading world, mobile cloud computing Such as Amazon Web Services, Google App Engine and Windows Azure grows to high level in computing technology of IT organizations and developers. Similarly, it was applied in all mobile phone platforms. This research paper present the applicability of MCC in various domains like mobile learning, commerce, healthcare and social Medias with energy consumption. It further identifies research gaps of vital challenges of MCC and effective resource utilization scale. Also, this research work explains an efficient resource allocation in the MCC through task distribution. The optimal Energy efficient method for mobile cloud environment has been discussed. However, there is a lack of optimization (i.e. where $\mathrm{T}_{\text {delay }}$ is the delay time, $\mathrm{N}$ is the length of data and $\mathrm{R}$ is

both power and execution time of optimization) in the mobile cloud data centers. The developed approach can facilitate and sustains a more green energy system. The upcoming research work in MCC can be focused on the limited resources of mobile devices and sensors in MCC ecosystems. Additionally, in this work the optimal criteria such as policies, execution time, energy conservation and cost are focused.

- Execution time: offloading will improve energy consumption.

- Energy: Previous data is supplied with ecological parameters to prioritize energy saving

- Execution time, energy and cost: It means cost on demand basis and so offloading decision could be manipulated with CPU time and battery power.

Power conservation is also done by means of the code offloading mechanism in MCC. The projected model results in the consideration measure $s$ of energy savings by the proposed OEAS algorithm.

\section{CONCLUSION}

This paper highlighted an energy efficient resource allocation management scheme accompanied by minimal power consumption by means of the enthalpy OEAS algorithm. The paper identified and located the various issues of mobile cloud and resolve issues of managing resource activity in an efficient manner. Several different iterative measures were found out for each activity and derived from the earlier enthalpy values, it finalize the value of the OEAS algorithm. This method prioritized the activities based on the maximum outcomes and through which, the resources were allocated efficiently and in an effective manner. The implementation of the suggested algorithm runs on a MATLAB platform. The energy consumption and execution time of the proposed scheme were considerably lowered. These findings rendered the proposed scheme as a viable and precious procedure for resource allocation optimization. The future work can be enhanced by altering other resource constraints as Input / Output, Memory and CPU.

\section{REFERENCES}

1. Foster, Y. Zhao, I. Raicu, and S. Lu, "Cloud computing and grid computing 360-degree compared," In Proceedings of Grid Computing Environments Workshop (GCE), IEEE, Austin, TX, 2008, pp. 1-10.

2. K. Kumar and Y. Lu, "Cloud computing for mobile users: Can offloading computation save energy?," Computer., 2010, Vol. 43(4), pp. 51-56.

3. Aepona, "White paper, mobile cloud computing solution brief," November 2010.

4. W. Jia, H. Zhu, Z. Cao, L. Wei and X. Lin, "SDSM: A secure data service mechanism in mobile cloud computing," IEEE Conference on Computer Communications Workshops (INFOCOM WKSHPS), Shanghai, China, 2011.

5. J. H. Christensen, "Using RESTful web-services and cloud computing to create next generation mobile applications," In Proceedings of the 24th ACM SIGPLAN conference companion on Object oriented programming systems languages and applications, Orlando, FL, 2009, pp. 627-634 
6. J. Oberheide, K. Veeraraghavan, E. Cooke, J. Flinn and F. Jahanian, "Virtualized in-cloud security services for mobile devices," In Proceedings of the First Workshop on Virtualization in Mobile Computing, Breckenridge, CO, 2008, pp. 31-35.

7. M. Reza Rahimi, J. Ren, C. H. Liu, A. V. Vasilakos and V. Nalini, "Mobile Cloud Computing: A Survey, State of Art and Future Directions," Mobile. Netw. Appl., 2014, Vol. 19(2), pp. 133-143.

8. O. A. Ben-Yehuda, M. Ben-Yehuda, A. Schuster, and D. Tsafrir "The Resource-as-a-Service (RaaS) cloud," in HotCloud '11: 4th USENIX Workshop on Hot Topics in Cloud Computing, Boston, MA, 2012, pp. 1-18,

9. CISCO, the Internet of things: How the Next Evolution of the Internet is Changing Everything, CISCO White Paper, 2011.

10. E. Ahmed, A. Gani, M. K. Khan, R. Buyya and S. U. Khan, "Seamless application execution in mobile cloud computing: Motivation, taxonomy, and open challenges," J. Netw. Comput. Appl., 2015, Vol. 52, pp. 154-172.

11. H. Viswanathan, E. K. Lee, I. Rodero and D. Pompili, "Uncertainty-aware autonomic resource provisioning for Mobile cloud computing," IEEE. T. Parall. Distr., 26(8), pp. 2363-2372, 2015.

12. O. Munoz Medina, A. Pascual-Iserte and J.Vidal, "Optimization of radio and computational resources for energy efficiency in latency constrained application offloading," IEEE. T. Veh. Technol., 2015, Vol. 64(10), 4738-4755.

13. M. Satyanarayanan, P. Bahl, R. Caceres and N. Davies, "The case for VM-based cloudlets in mobile computing," IEEE. Pervas. Comput., 2009, Vol. 8(4), pp. 14-23.

14. Y. Zhang, D. Niyato and P. Wang, "Offloading in mobile cloudlet systems with intermittent connectivity," IEEE. T. Mobile. Comput., 2015, Vol. 14(12), pp. 2516-2529.

15. T. Liu, F. Chen, Y. Ma, and Y. Xie, "An energy-efficient task scheduling for mobile devices based on cloud assistant," Future. Gener. Comp. Sy., 2016, Vol. 61, pp. 1-12.

16. Y. Liu, and M. J. Lee, “An adaptive resource allocation algorithm for partitioned services in mobile cloud computing," IEEE Symposium Service-Oriented System Engineering (SOSE), San Francisco Bay, CA, 2015, pp. 209-215.

17. L. Tang and H. Chen, "Joint pricing and capacity planning in the iaas cloud market," IEEE. T. Cloud. Comput., 2017, Vol. 5(1), pp. 57-70.

18. Y. Liu and M. J. Lee, "Security-aware resource allocation for mobile cloud computing systems," $24^{\text {th }}$ International Conference on Computer Communication and Networks (ICCCN), Las Vegas, NV, pp. 1-8, 2015.

19. N. C. Luong, P. Wang, D. Niyato, Y. Wen and Z. Han, "Resource management in cloud networking using economic analysis and pricing models: a survey," IEEE. Commun. Surv. Tut., 2017, Vol. 19(2), pp. 954-1001.

20. S. S. Manvi and G. K. Shyam, "Resource management for Infrastructure as a Service (IaaS) in cloud computing: A survey", J. Netw. Comput. Appl., 2014, Vol. 41, pp. 424-440.

21. D. Merezeanu, G. Vasilescu and R. Dobrescu, "Context-aware Control Platform for Sensor Network Integration in IoT and Cloud," Stud. Inform. Control., 2016, Vol. 25(4), 489-498.

22. M. A. Mohammed and R. A. K. Hasan, "Particle Swarm Optimization for facility layout problems FLP - A comprehensive Study," IEEE 13th International Conference on Intelligent Computer Communication and Processing, (ICCP), Cluj-Napoca, Romania, 2017, pp. 1-7.

23. L. Tawalbeh, Y. Jararweh, F. Ababneh and F. Aldosari, "Large-scale cloudlets deployment for efficient mobile cloud computing," J. Netw., 2015, Vol.10(1), pp.70-76.
Mrs. M. R. Sudha is currently pursuing $\mathrm{Ph}$. D

research under the guidance of Dr. C. P. Sumathi

in the area of Cloud and Mobile Cloud Computing. She is currently working as an Assistant Professor, Department of Computer Applications, Faculty of Science and Humanities at the SRM Institute of Science and Technology (formerly known as SRM University, Chennai, India) is doing research in an IOT, Green Energy in Cloud Data Centre Cloud and Mobile Cloud Computing. She is having 18 years teaching experience in the Department of Computer Science. She has been working as Lecturer cum Head of the Department of Computer Science in N.M. S Sermathai Vasan College for Women, Madurai, India. She is a life time member of the International Association of Engineers, (IAENG) since 2018. Her teaching interests include Operating System and Object oriented programming in $\mathrm{C}++$.

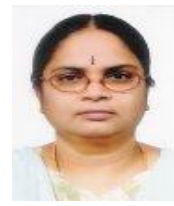

Dr. C. P. Sumathi pursued her Ph.D from University of Madras. She is currently the Associate Professor and Head, Research Department of Computer Science, SDNB Vaishnav College for Women, Chrompet, Chennai. She has got 27 years of teaching experience. She has guided more than $10 \mathrm{Ph}$. D Scholars. She has published more than 60 research papers in reputed international journals including Scopus, Thomson Reuters (SCI \& Web of Science), conferences including IEEE and it's also available online. Her main research work focuses on Fingerprinting Algorithms, Image Processing, Data Mining, Information Security, Cloud and Mobile Cloud Computing.

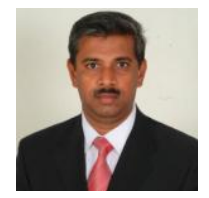

Dr. A. Saravanakumar pursued his $\mathrm{Ph}$. D from National Renewable Energy Fellowship Programme Sponsored by Ministry of New and Renewable Energy, Govt. of India through Centre for Energy Studies, IIT, Delhi for the Department of Bio Energy, School of Energy, Environment and Natural Resources, Madurai Kamaraj University, Madurai. He is having 18 years R \& D experience in the field of Renewable Bio Energy. He is currently working as an Assistant Professor, Department of Physics, Easwari Engineering College, Ramapuram, Chennai. He got the Certificate of Outstanding Contribution in Reviewing Award of Applied Energy, Elsevier, Amsterdam, The Netherlands. His main research work focuses on Renewable Energy based Cloud Data Center and Bio Energy based Power Plants. 